\title{
Resection of rectal cancer resembling submucosal tumor that was preoperatively diagnosed with endoscopic ultrasound- guided biopsy
}

Akimitsu Tanio, Hiroaki Saito ${ }^{*}$ D, Keigo Ashida, Shouichi Urushibara, Manabu Yamamoto, Naruo Tokuyasu, Teruhisa Sakamoto, Soichiro Honjo, Yoshihiko Maeta and Yoshiyuki Fujiwara

\begin{abstract}
Background: Colorectal cancer (CRC) resembling submucosal tumor (SMT; CRC/SMT) is very rare. Because its biopsy is challenging, accurate preoperative diagnosis is also very rare.

Case presentation: A 55-year-old woman with a high serum carcinoembryonic antigen level underwent a computed tomography colonoscopy, which showed extrinsic rectum compression. A coronal magnetic resonance image showed a 4-cm low-intensity tumor between her rectum and sacrum. Endoscopic ultrasound (EUS) showed a 30-mm low-echoic lesion originating from the rectum. Pathological examination of specimen obtained with EUSguided fine-needle aspiration biopsy (EUS-FNAB) revealed adenocarcinoma. Immunohistochemical staining showed the tumor to be positive for both CK20 and CDX2 and negative for CK7, indicating that it was a rectal cancer. We performed a laparoscopy-assisted low-anterior resection with dissection of the regional lymph nodes after eight chemotherapy cycles. Macroscopically, tumor was completely covered by normal rectal mucosa, but showed a 2-mm bulge on the mucosa. Histological examination revealed a moderately differentiated adenocarcinoma, mainly located at the subserosal layer and severely invaded to lymphatic and blood vessels. The mucosal layer was not exposed to the cancer components, and her postoperative course was uneventful.
\end{abstract}

Conclusion: EUS-FNAB was useful in preoperative accurate diagnosis of this very rare tumor. We also review the literature and discuss CRC/SMT.

Keywords: Colorectal cancer, Endoscopic ultrasound-guided fine needle aspiration biopsy, Submucosal tumor

\section{Background}

Submucosal tumor (SMT) usually arises from tissue in the wall of digestive tract, and its surface is therefore covered with normal mucosa in most cases. In contrast, gastrointestinal (GI) carcinomas arise from the epithelium, and most of their mucosal surfaces typically consist of cancerous tissue. SMT-like growth is an unusual presentation for GI carcinomas, especially in colorectal cancer (CRC). We herein report a rare case of rectal cancer resembling SMT (CRC/SMT) in which endoscopic

\footnotetext{
*Correspondence: sai10@med.tottori-u.ac.jp

Division of Surgical Oncology, Department of Surgery, School of Medicine,

Tottori University Faculty of Medicine, 36-1 Nishi-cho, Yonago 683-8504, Japan
}

(c) The Author(s). 2017 Open Access This article is distributed under the terms of the Creative Commons Attribution 4.0 International License (http://creativecommons.org/licenses/by/4.0/), which permits unrestricted use, distribution, and reproduction in any medium, provided you give appropriate credit to the original author(s) and the source, provide a link to the Creative Commons license, and indicate if changes were made. ultrasound-guided fine needle aspiration biopsy (EUSFNAB) was useful in accurate preoperative diagnosis. We also review the literature and discuss CRC/SMT.

\section{Case presentation}

An asymptomatic 55-year-old woman underwent a detailed GI tract examination, as she was found to have high serum carcinoembryonic antigen (CEA, $26.6 \mathrm{ng} / \mathrm{ml}$ ) at a local hospital. She had no event in her past or family history, especially for colorectal neoplasm or other malignancy. Physical examination and blood chemical examination were within normal ranges except for high serum CEA level. Computed tomography (CT) colonoscopy revealed extrinsic rectum compression (Fig. 1a). A

\section{SpringerOpen}



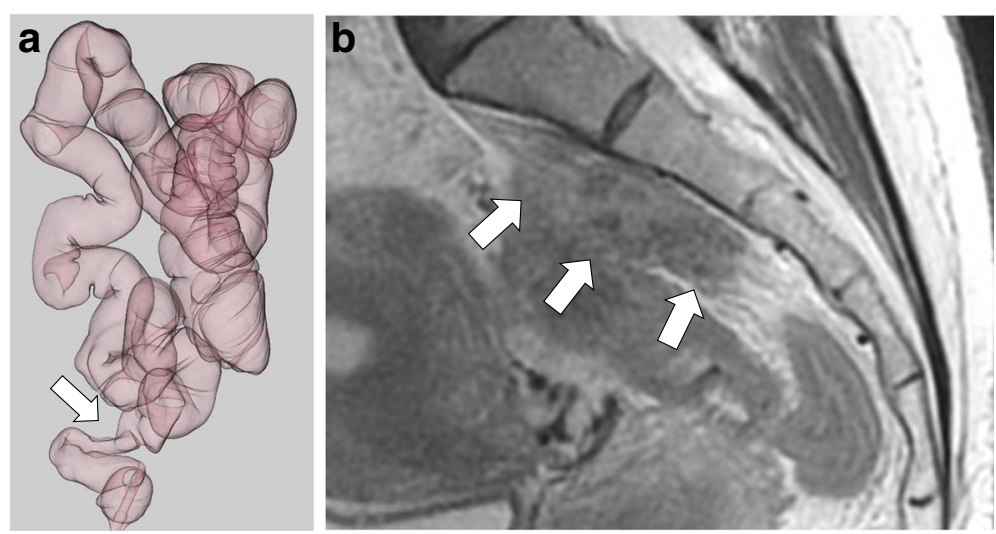

Fig. 1 a CT colonoscopy revealed extrinsic compression of rectum. b Coronal magnetic resonance imaging (MRI) showed 4-cm low-intensity tumor between rectum and sacrum

coronal magnetic resonance image (MRI) showed a 4-cm low-intensity tumor between the rectum and sacrum (Fig. 1b); the patient was referred to our hospital for further evaluation of this tumor. The 18-FDG positron emission tomography (PET) and CT scans indicated high 18-FDG uptake in the primary tumor (SUVmax: 3.34; Fig. 2a), with enlarged para-aortic lymph node (SUVmax: 2.58; Fig. 2b) and anterosuperior segment of right hepatic lobe (SUVmax: 2.74; Fig. 2c). Colonoscopy showed extrinsic rectum compression, but no change in her rectal mucosa (Fig. 3a). Pathological examination of rectum biopsy specimen showed normal rectal mucosa. Endoscopic ultrasound (EUS) showed a 30-mm, low-echoic lesion originating from the rectum (Fig. 3b). Pathological examination of biopsied specimen obtained with EUS-guided fine-needle aspiration biopsy (EUS-FNAB) revealed adenocarcinoma. Immunohistochemical staining showed that the specimen was positive for both cytokeratin 20
(CK20; Fig. 3c) and caudal-type homeobox 2 (CDX2; Fig. 3d) and negative for cytokeratin-7 (CK7), which indicated that it had originated from rectal cancer. We planned to perform low-anterior resection with dissection of the regional lymph nodes and an enlarged para-aortic lymph node under the diagnosis of rectal cancer and possible metastasis to liver and para-aortic lymph node, followed by resection of possible liver metastasis after postoperative chemotherapy. Because of a possibility that tumor had invaded to the sacrum, however, we performed neoadjuvant chemotherapy with capecitabine and oxaliplatin. The tumor shrank by $38 \%$ after 8 courses of chemotherapy, and we performed a laparoscopy-assisted low-anterior resection with dissection of the regional lymph nodes as planned. Although we confirmed direct invasion of the tumor to hypogastric nerve during the surgery, the tumor did not invade to the sacrum. An enlarged para-aortic lymph
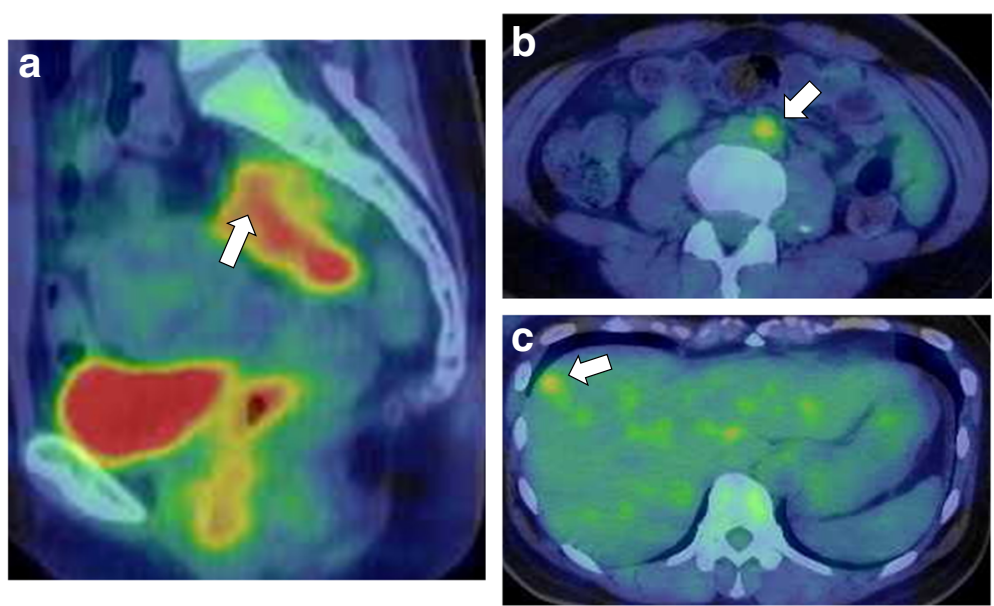

Fig. 2 The 8-FDG positron emission tomography (PET) and CT scans indicate high 18-FDG uptake in (a) the primary tumor (SUVmax: 3.34); (b) enlarged para-aortic lymph node (SUVmax: 2.58); and (c) anterosuperior segment of right hepatic lobe (SUVmax: 2.74) 

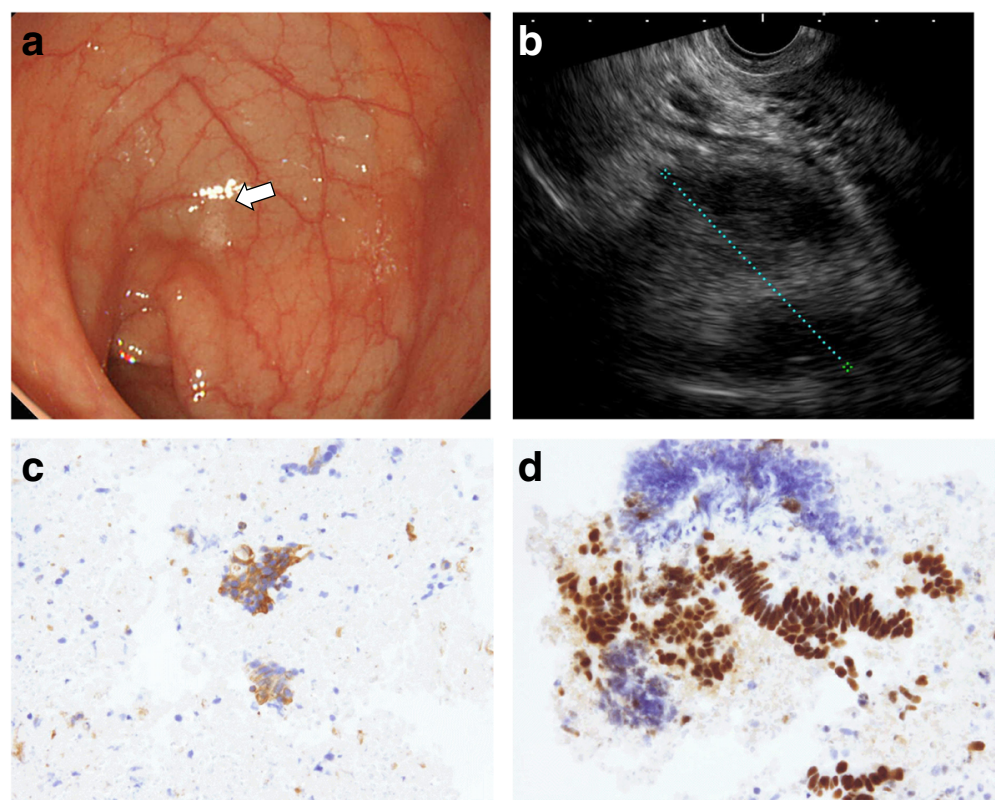

Fig. 3 a Colonoscopy also showed extrinsic compression of rectum, but no change in rectal mucosa. b Endoscopic ultrasound (EUS) showed a 30-mm low echoic lesion originating from rectum. Immunohistochemical staining of biopsy specimen obtained by EUS-FNAB showed that tumor was positive for both (c) CK20 and (d) CDX2

node was also removed. Macroscopically, the tumor was completely covered by normal rectal mucosa (Fig. 4a), although there was a 2-mm bulge of elevated lesion on the mucosa (Fig. 4b). Histologically, the tumor was composed from moderately differentiated adenocarcinoma (Fig. 5a) and was mainly located at subserosal region with severe invasion to lymphatic and blood vessels (Fig. 5b). However, the mucosal surface showed no exposure of the cancer component (Fig. 5c). Metastases were found in two lymph nodes of mesorectum (\#251) and in one lymph node along the superior rectal artery (\#252), although there was no metastasis in the para-aortic lymph node dissected. The postoperative course was uneventful. The patient is currently undergoing chemotherapy for liver metastasis in the outpatient clinic of our hospital.

\section{Discussion}

Carcinomas of the digestive tract arise from the mucosal epithelium; most of their mucosal surfaces consist of cancerous tissues. Although cancer cells then infiltrate both horizontally and vertically, the dominant direction depends on the nature of the cancer cells. Some intestinal cancers dominantly infiltrate in a vertical direction without massive invasion along the horizontal plane. As a result, the internal intestinal surfaces over these tumors are mostly covered with normal mucosa, and therefore manifest as SMTs. This type of tumor is sometimes called a carcinoma resembling an SMT; and although it is extremely rare, has been reported in the esophagus [1], stomach [2], and colon and rectum [3, 4]. A report that reviewed 70 reported cases of CRC/SMT
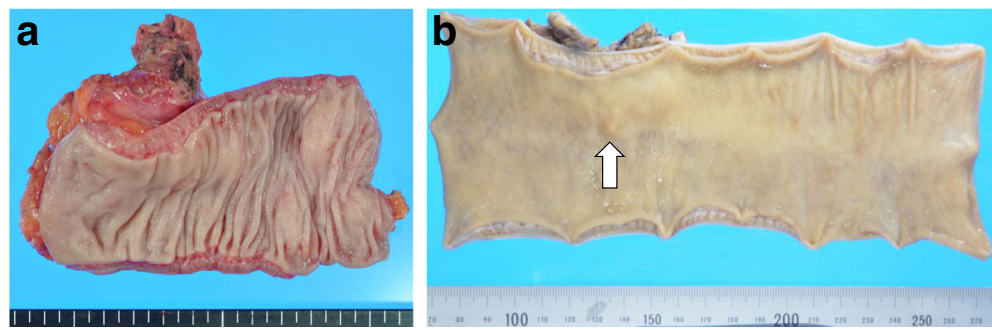

Fig. 4 a Macroscopically, the tumor was completely covered by normal rectal mucosa. b The formalin-fixed resected specimen showed a 2-mm bulge above the mucosa level 
b
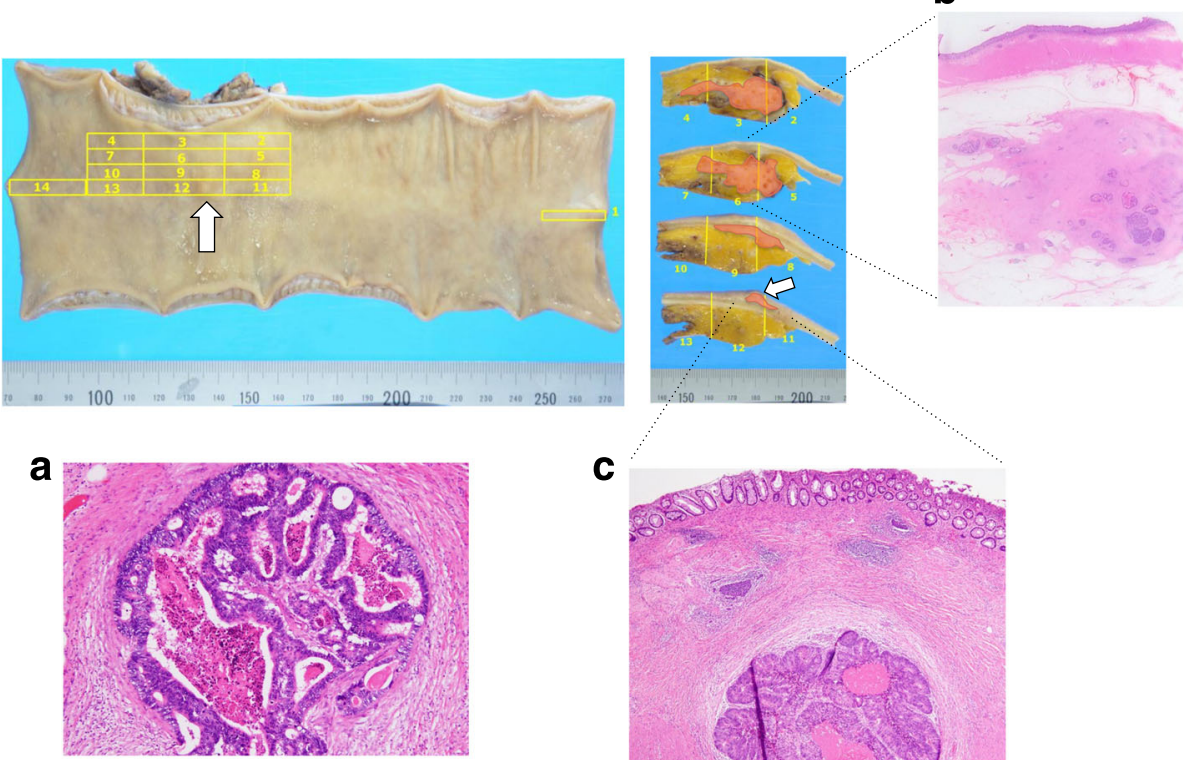

Fig. 5 a Histologically, the tumor was composed of moderately differentiated adenocarcinoma. b The carcinoma was mainly located at subserosa and had severely invaded to lymphatic and blood vessels. c Adenocarcinoma was observed in the submucosal layer and the mucosal surface showed no exposure of the cancer component

found it to be characterized by small tumor size, high rates of poorly differentiated adenocarcinoma or mucinous adenocarcinoma, and invasiveness (including high rates of lymph node metastasis and lymphatic vessel invasion) [4].

Two points make our case different from previously reported CRC/SMT. Firstly, the tumor was completely covered by normal rectal mucosa in our case. Most tumors reported previously showed at least faint mucosal changes, such as erosion and ulceration, which are microscopically consistent with exposure of carcinoma cells. Because most of the cancer on our case was located subserosally with no exposure of cancer cells on mucosal surface, metastatic rectal cancer was considered. In this regard, immunohistochemical staining of the biopsied specimen showed the tumor was positive for CK20 and negative for CK7, indicating the strong possibility that our patient had primary rectal cancer. To the best of our knowledge, only four cases (including the present case) have been reported of CRC/SMT that were completely covered with normal rectal mucosa (Table 1) [5-7]. Why no cancer cells were exposed on the mucosal layer remains unclear. Cancer may arise from ectopic mucosal cells in the rectal wall. Further investigations are required to show the detailed mechanism.

Secondly, our case clearly showed that EUS-FNAB is useful for accurate preoperative diagnosis of CRC/SMT. EUS-FNAB was recently shown to be useful in accurate preoperative diagnosis of gastrointestinal stromal tumor (GIST) [8] and pancreatic cancer [9]. Previous reports have shown the difficulty of preoperative CRC/SMT diagnosis, because of the difficulty of obtaining biopsy specimens. Therefore, EUS-FNAB should be considered to make accurate preoperative SMT diagnoses in colon

Table 1 Reported cases of colorectal cancer resembling SMT in which tumor was completely covered with normal colorectal mucosa

\begin{tabular}{|c|c|c|c|c|c|c|c|c|c|c|c|c|}
\hline Case & Year & $\begin{array}{l}\text { Age } \\
\text { (years) }\end{array}$ & Gender & Location $^{\text {a }}$ & $\begin{array}{l}\text { Size } \\
(\mathrm{cm})\end{array}$ & $\begin{array}{l}\text { Endoscopic } \\
\text { findings }\end{array}$ & $\begin{array}{l}\text { Depth of } \\
\text { invasion }^{b}\end{array}$ & $\begin{array}{l}\text { Histologic } \\
\text { diagnosis of } \\
\text { biopsy specimen }\end{array}$ & $\begin{array}{l}\text { Histologic } \\
\text { diagnosis of } \\
\text { resected specimenc }\end{array}$ & $\begin{array}{l}\text { Lymph } \\
\text { node } \\
\text { metastasis }\end{array}$ & $\begin{array}{l}\text { Distant } \\
\text { metastasis }\end{array}$ & Reference \\
\hline 1 & 2005 & 57 & M & $\mathrm{Rb}$ & 10 & None & A & Unknown & Muc & Absent & Unknown & 5 \\
\hline 2 & 2011 & 82 & M & $S$ & 8 & None & SS & Normal mucosa & Muc & Absent & Absent & 6 \\
\hline 3 & 2014 & 79 & M & Ra & 2.6 & Erosion & SE & Not performed & Mod & Absent & Absent & 7 \\
\hline 4 & 2017 & 55 & $\mathrm{~F}$ & RS & 4 & None & SS & Normal mucosa & Mod & Present & $\begin{array}{l}\text { Liver } \\
\text { metastasis }\end{array}$ & Our case \\
\hline
\end{tabular}

aLocation. $R a$ upper rectum, $R b$ lower rectum, $R S$ rectosigmoid, $S$ sigmoid colon

${ }^{b}$ Depth of invasion. $A$ adventitia, SE serosa, SS subserosa

'Histologic diagnosis of resected specimen. Mod moderately differentiated adenocarcinoma, Muc mucinous carcinoma 
and rectum. To our knowledge, our case is the first to use EUS-FNAB for an accurate preoperative diagnosis of CRC/SMT.

\section{Conclusion}

We should keep in mind that colorectal cancer can present with SMT-like growth. Furthermore, EUS-FNAB is useful for preoperative accurate diagnosis of this rare colorectal cancer.

Received: 3 April 2017 Accepted: 11 July 2017

Published online: 26 July 2017

\section{Authors' contributions}

AT contributed to the drafting of the manuscript. KA, SU, MY, NT, TS, SH, and YM contributed to the critical revision of the manuscript. HS and YF contributed to the final approval of the manuscript. All authors read and approved the final manuscript.

\section{Consent for publication}

Consent for publication was obtained from the patients.

\section{Competing interests}

The authors declare that they have no competing interests.

\section{Publisher's Note}

Springer Nature remains neutral with regard to jurisdictional claims in published maps and institutional affiliations.

Received: 3 April 2017 Accepted: 11 July 2017

Published online: 26 July 2017

\section{References}

1. Kishino T, Yamaguchi Y, Yamagishi T, Hashimoto T, Kyomasu Y, Mori H, et al. Submucosal tumor (SMT)-like esophageal squamous cell carcinoma with gastric metastasis. Hepatogastroenterology. 2000;47:1581-4.

2. Ohara N, Tominaga O, Uchiyama M, Nakano H. A case of advanced gastric cancer resembling submucosal tumor of the stomach. Jpn J Clin Oncol. 1997;27:423-6.

3. Shinagawa T, Ishihara S, Nozawa H, Murono K, Nishikawa T, Otani K, et al. Small colorectal cancers resembling submucosal tumor with massive submucosal invasion and lymph node metastasis: A report of two cases and review of the literature. Clin Res Hepatol Gastroenterol. 2016;41:e19-e23.

4. Nakajima T, Kamano T, Shibasaki K, Watanabe K, Meguro H, Tomiki Y, et al. Colon carcinoma resembling submucosal tumor: report of a case and review of the literature. Int J Gastrointest Cancer. 2005:36:155-61.

5. Kameda K, Matsuda G, Nakashima M, Kubo A, Takekawa Y, Kato K, et al. A case of mucoid carcinoma of rectum presented as a submucosal tumor which was discovered by elevated value of CEA. J Japan Surg Assoc. 2005; 66:1985-9.

6. Sato K, Kojima Y, Matsuno Y, Watanabe Y. A case of mucoid carcinoma of the sigmoid colon with features of a submucosal tumor. J Japanese Society Gastroenterol. 2011;108:88-94.

7. Shiraiwa S, Taniwaki S, Imamura T, Sou H, Shimokobe T, Morimitsu Y. A case of rectal cancer presented as submucosal tumor without the continuation with the mucous membrane. J Jpn Surg Assoc. 2014;75:749-53.

8. Mekky MA, Yamao K, Sawaki A, Mizuno N, Hara K, Nafeh MA, et al. Diagnostic utility of EUS-guided FNA in patients with gastric submucosal tumors. Gastrointest Endosc. 2010;71:913-9.

9. Duskova J, Krechler T, Dvorak M. Endoscopic ultrasound-guided fine needle aspiration biopsy of pancreatic lesions. An 8-year analysis of single institution material focusing on efficacy and learning progress. Cytopathology. 2016;28:109-15.

\section{Submit your manuscript to a SpringerOpen ${ }^{\bullet}$ journal and benefit from:}

- Convenient online submission

Rigorous peer review

- Open access: articles freely available online

- High visibility within the field

- Retaining the copyright to your article

Submit your next manuscript at $\boldsymbol{\nabla}$ springeropen.com 